\title{
An Analysis of VoIP Application in Fixed and Mobile WiMAX Networks
}

\author{
Pranav Gangadhar Balipadi ${ }^{\mathrm{a}}$, J Sangeetha ${ }^{\mathrm{b}}$
}

${ }^{a b}$ Department of Information Science \& Engineering, PES Institute of Technology, Bangalore - 560085, India

\begin{abstract}
WiMAX is a wireless broadband technology, which promises maximum coverage area and high data rates. WiMAX provides last mile connectivity. This network defines two working models such as fixed WiMAX and mobile WiMAX. The main aim of this paper is to compare and also analyze the performance of the VoIP application over fixed and mobile WiMAX, with respect to various codecs such as G.711, G.723.1ar5.3, G.726ar24, G.728ar16 and G.729. We have considered few QoS parameters such as average end-to-end delay, throughput, average jitter, average one-way delay and average MOS. From the obtained result, codec G.711 and G.726ar24 performs better when compared to other codecs in both fixed and mobile WiMAX network.
\end{abstract}

Index Terms: Fixed WiMAX, Mobile WiMAX, VoIP application.

(C) 2016 Published by MECS Publisher. Selection and/or peer review under responsibility of the Research Association of Modern Education and Computer Science

\section{Introduction}

The IEEE 802.16 standard is also known as worldwide interoperability for Microwave Access (WiMAX).It is one of the broadband wireless technologies, which was developed in the year 1998. It is a Wireless Metropolitan Area Network (WMAN). WiMAX network provides better Quality of Service (QoS) to residential and enterprise customers. WiMAX is an alternative to wired technologies such as cable modems and Digital Subscribers Line (DSL). It also has the ability to provide better service in areas where wired infrastructure is difficult to reach. WiMAX solves last mile connectivity problem which means WiMAX provides sufficient bandwidth for the device which is connected at the end of connection. It offers high throughput, large network coverage, flexible QoS support and extensive security [1]. Its frequency range is 10$66 \mathrm{GHz}$ and maximum bandwidth of $120 \mathrm{Mbps}$ with maximum transmission range up to $50 \mathrm{Km}$ [2]. The IEEE 802.16 working group consists of two types of models, fixed WiMAX (i.e. IEEE 802.16d) and mobile WiMAX $[3,4]$ (i.e. IEEE 802.16e).

Recently, many researchers have focused on using VoIP application in WiMAX networks. In [5], the authors

* Corresponding author. Tel.: +919916719937

E-mail pranavgb29@gmail.com 
used Non Ad-Hoc (NOAH) routing algorithm in WiMAX network. They have analyzed the VoIP application with respect to G.723 and G.729 codecs. Only 6 nodes were considered for analysis and comparison. The different QoS parameters considered are throughput, average jitter and average end-to-end delay. From the result, the authors have concluded that the G.723 is a best codec for Best Effort (BE) service flow and G.711 and G.729 gives high throughput. In [6], the authors have analyzed the VoIP application with respect to G.711 and G.729 codecs. Only 80 nodes were considered for analysis and comparison. The different QoS parameter considered are average jitter, average end-to-end delay and throughput. From the result, the authors have concluded that G.729 is better than G.711.

Our contribution to the paper is that we are considering the VoIP application in WiMAX network (i.e. both fixed and mobile WiMAX) with the codecs such as G.711, G.723.1ar5.3, G.726ar24, G.728ar16 and G.729. We have considered few QoS parameters such as: average end-to-end delay, throughput, average jitter, average one-way delay and average Mean Opinion Scale (MOS) to analyze the performance of the above mentioned codecs. In our simulation, we have considered 250 nodes. The organization of the paper is as follows: the fixed WiMAX and mobile WiMAX are explained in detail in section 2 and section 3 respectively. In section 4 we have differentiated fixed and mobile WiMAX technologies. The VoIP applications are discussed in section 5. The result and discussion is brought in section 6 . We conclude our work in section 7 .

\section{Fixed WiMAX}

The IEEE 802.16d standard is also known as fixed WiMAX which is as shown in Fig 1. The fixed WiMAX supports only fixed application. It is very robust against multi-path propagation because it uses Orthogonal Frequency Division Multiplexing (OFDM). OFDM belongs to transmission scheme called multicarrier modulation, which is based on an idea where the high data stream are divided into several parallel lower rate streams, and each of these streams are modulated on separate carriers and these carriers are called subcarriers. In OFDM where the subcarriers are selected in such a way that they are all orthogonal to each other, which decreases overlapping subcarrier channels. Fixed WiMAX can work as a point-to-multipoint with the transmission data rate of $1 \mathrm{Mbps}$ to $75 \mathrm{Mbps}$ at a transmission distance over $50 \mathrm{~km}$. It operates in $3.5 \mathrm{GHz}$ and $5.8 \mathrm{GHz}$ spectrum bands [7].

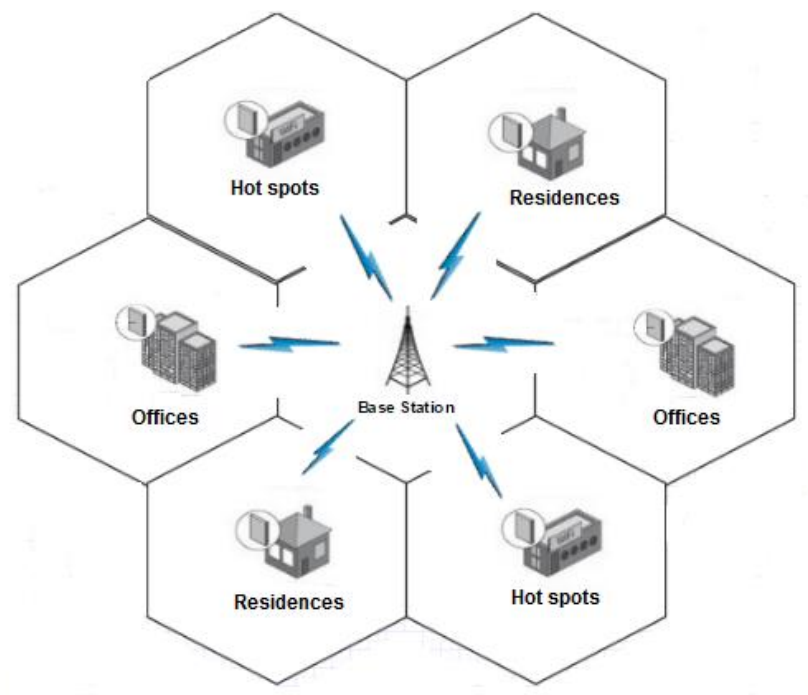

Fig.1. Fixed WiMAX 


\section{Mobile WiMAX}

The IEEE 802.16e standard is also known as mobile WiMAX which is as shown in Fig 2. The mobile WiMAX is an amendment to fixed WiMAX by adding mobility [8]. The mobile WiMAX supports mobile, portable, fixed and nomadic applications. Mobile WiMAX uses the Scalable OFDMA (SOFDMA) for improving the multipath performance in non-line-of-sight [9]. In SOFDMA scalability is backed by modifying the size of Fast Fourier Transform (FFT) size. Large and small FFT channels are provided to wider and lower bandwidth channels respectively [10].Here the sub-carrier spacing does not depend on bandwidth, and the volume of sub-carriers scale with bandwidth. Mobile WiMAX [3, 4] works on the licensed spectrum allocated in the $2.3 \mathrm{GHz}, 2.5 \mathrm{GHz}$ and $3.5 \mathrm{GHz}$ frequency bandwidth [7].

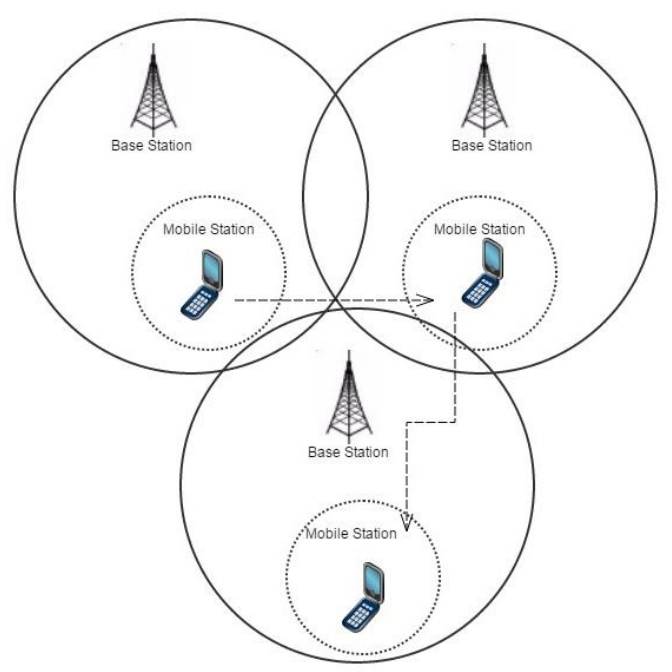

Fig.2. Mobile WiMAX

\section{Comparison of fixed and mobile WiMAX}

The IEEE 802.16d uses the Single Input Single Output (SISO) technique for data transmission whereas the IEEE 802.16e uses the Multiple Input Multiple Output (MIMO) technique for data transmission. The major difference between fixed and mobile WiMAX is that the mobile WiMAX helps in mobility management.

Table 1. Difference between Fixed and Mobile WiMAX

\begin{tabular}{lll}
\hline Feature & Fixed WiMAX & Mobile WiMAX \\
\hline IEEE Standard & $802.16 \mathrm{~d}$ & $802.16 \mathrm{e}$ \\
Release & June 2004 & December 2005 \\
Multiplexing & OFDM & SOFDMA \\
FFT Size & 256 & 512,1024 \\
Hand Offs & None & Yes \\
Frequency Bandwidth & $2-11 \mathrm{GHz}$ & $2-3.5 \mathrm{GHz}$ \\
Services Supported & Fixed, nomadic and portable & Mobility, Fixed and nomadic \\
Coverage area & $3-5$ miles & 2 miles \\
Transmission technique & SISO & MIMO \\
QoS Classes Supported & UGS, rtPS, nrtPS, and BE & UGS, rtPS, nrtPS, ertPS and BE \\
\hline
\end{tabular}


The other difference is that fixed WiMAX uses the OFDM for its PHY layer whereas Mobile WiMAX uses SOFDMA [10]. The IEEE 802.16d standard uses the QoS classes such as Unsolicited Grant Service (UGS), real time Polling Service (rtPS),non-real time Polling Service (nrtPS) and Best Effort (BE) whereas mobile WiMAX uses the same classes of fixed WiMAX but it adds an additional QoS class called as extended real time Polling Service (ertPS) $[11,28]$. The comparisons of both fixed and mobile WiMAX are shown in the Table 1.

\section{VoIP Application}

With fast growing Internet, there is a demand for VoIP application. The VoIP applications are used to carry voice over Internet, also the cost is less compared to traditional telephone lines [12]. VoIP application uses different varieties of signalling protocols such as: H.323, Session Initiation Protocol (SIP), Real-time Transport Protocol (RTP) and Real-time Transport Control Protocol (RTCP). Among these protocols H.323 and SIP are generally used for call signalling and call management purposes [13]. In our study, we have used H.323 signalling protocol.

H.323 is a standard defined by the International Telecommunication Union-Telecommunication Standardization Sector (ITU-T) [14], for the transmission of real-time audio, video and data over packet switched networks. It specifies the components, protocols and procedures for multimedia application. The main goal of H.323 signalling protocol is to communicate and operate with other multimedia networks. H.323 consists of the following frameworks such as: H.245 for control signalling, H.225.0 for establishment of connection, H.332 for large conferences, H.450.1, H.450.2 and H.450.3 for supplementary services, H.235 for security purpose and H.246 for circuit switched services [15].

Codec is a term used for the word coder-decoder [16]. Codecs are used to convert analog audio signal into compressed digital signals and vice versa [17]. Various codecs are used for transmission of VoIP application. The following codecs are implemented by the H.323 signalling protocol and they have been listed below.

- Audio codecs: G.711, G.723, G.726, G.728, G.729 and G.729a.

- Text codecs: T.140

- Video codecs: H.261, H.263, H.264

In this study we have used codecs such as G.711, G.723.1ar5.3, G.726ar24, G.728ar16 and G.729.

\subsection{G.711}

G.711 is a standard codec with high bit rate about 64 Kbps [18, 19] and is also known as Pulse Code Modulation (PCM) of voice frequencies [20]. It is a public domain codec widely used in VoIP applications. This codec is used by the Public Switched Telephone Network (PSTN) and Integrated Services Digital Network (ISDN) lines and it gives better voice quality when used under VoIP application. G.711 employs logarithmic compression that compresses each 16 bit sample to 8 bit sample. It can be used for fax communication over IP networks [21].

\subsection{G.723.1ar5.3}

G.723 is one of the most efficient and licensed codec with highest compression ratio. It has two versions with distinct bit rates such as $5.3 \mathrm{Kbps}$ and $6.4 \mathrm{Kbps}$. For better quality of speech rate, the G.723 codec performs compression and decompression of $8 \mathrm{KHz}$ to 5.3 to $6.4 \mathrm{Kbps}$. For the low bit rate it uses the algorithm called as Algebraic Code Excited Linear Prediction (ACELP) [16]. This codec is widely used in applications such as audio, video, fax, telephony, speech and also used in VoIP applications. 


\subsection{G.726ar24}

G.726 is an ITU standard codec, which has transmission rates such as 16, 24, 32, and $40 \mathrm{Kbps}$. It is used for international trunks to save bandwidth. It is also used in Digital Enhanced Cordless Telephony (DECT) wireless phones. The G.726 uses Adaptive Differential pulse Code Modulation (AADPCM) [22]. The applications of G.726 are telephony, voice storage, multimedia \& video conferencing, digital circuit multiplication and networks and VoIP.

\subsection{G.728arl6}

G.728 speech codec was standardized by ITU-t in 1992. This codec operates on 16 bits speech signal at 8 $\mathrm{KHz}$ and produces a compressed bit stream with bit rate of $16 \mathrm{Kbps}$. It is based on Low Delay Codebook Excited Linear Prediction (LDCELP) compression technique [22]. G.728 rates as toll quality, so that voice quality is better than all codecs. It is used in satellite telephony, video conferencing and voice over cable application.

\subsection{G.729}

G.729 is an ITU standard codec and also industry standard licensed codec, it offers toll-quality speech at a reasonably low bit rate of $8 \mathrm{Kbps}$. This codec is based on the Conjugate-Structure Algebraic Code Excited Linear Prediction (CS-ACELP) algorithm [16]. This codec has low bandwidth requirements but provides good audio quality. The applications of this codec are fax, telephony, VoIP, Fax over Internet Protocol (FoIP), voice compression, imaging and video software for various applications.

\section{Result and discussion}

In this section, we are focusing on analyzing VoIP application [23] in fixed and mobile WiMAX network. In this study we have considered few QoS parameters such as: average end-to-end delay, throughput, average jitter and one-way delay and VoIP parameter Mean Opinion Score (MOS) and also considered up to 250 nodes for both fixed and mobile WiMAX network. We have used QualNet 7.1 network simulator. This simulator is used for creating and animating different network scenarios [24]. The different simulation parameters are shown in the Table 2.

Table 2. Simulation Parameters

\begin{tabular}{ll}
\hline Parameters & Value \\
\hline Transmission power & $20 \mathrm{~W}$ \\
Simulation area & $1500 \mathrm{~m} \mathrm{X} \mathrm{1500} \mathrm{m}$ \\
Number of nodes & $50,100,150,200$, and 250 \\
Radio type & 802.16 \\
Application type & VoIP \\
Simulation time & 100 seconds \\
\hline
\end{tabular}

Here, we have considered two different scenarios, one for fixed WiMAX and the other for mobile WiMAX. Both network scenarios are simulated for VoIP application. The different VoIP parameters are shown in the Table 3. 
Table 3. VoIP parameters.

\begin{tabular}{ll}
\hline Parameters & Value \\
\hline Multimedia Signalling Protocol & H323 \\
VoIP call Timeout & 60 \\
VoIP Connection Delay & 8 seconds \\
VoIP total loss probability & 2.07 \\
Jitter Buffer size(packets) & 30 \\
Average Talking Time & 20 seconds \\
Encoding Codecs & G.711,G.723.1ar5.3,G.726ar24,G.728ar16 \\
& and G.729 \\
\hline
\end{tabular}

\subsection{Average End-to-End Delay}

The average end-to-end delay is defined as the time taken by the bit of data to reach from source to destination node across the network, and it is measured in seconds. The graph for the analysis of average endto-end delay for fixed WiMAX is shown in Fig 3(a).

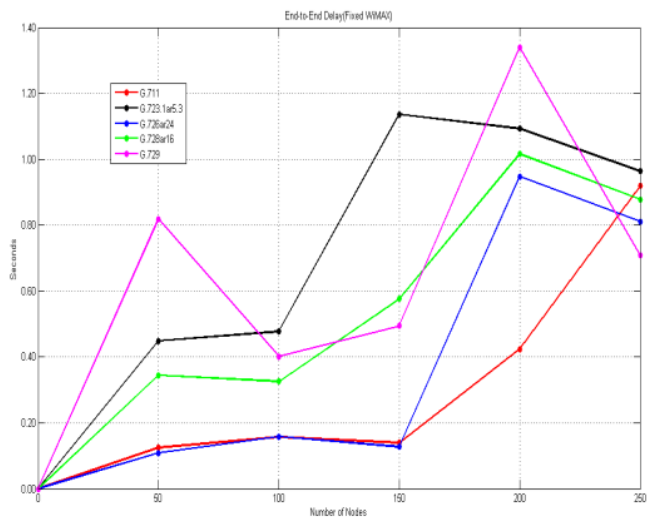

Fig.3. (a) Average end-to-end delay of Fixed WiMAX with respect to number of node

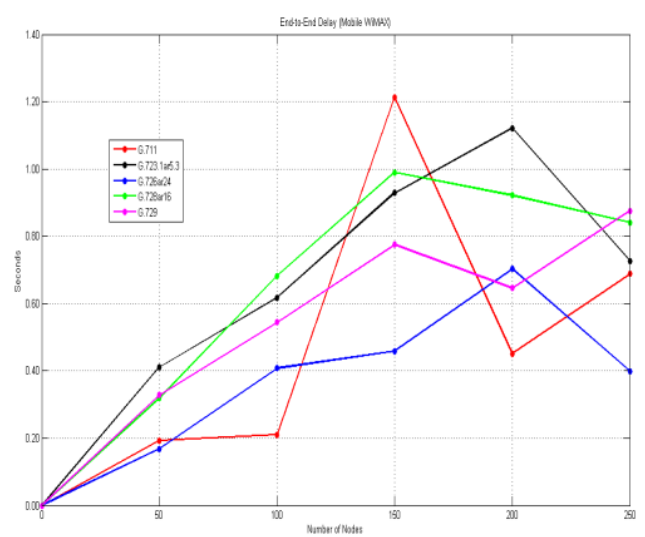

Fig.3. (b) Average end-to-end delay of Mobile WiMAX with respect to number of nodes 
In fixed WiMAX, which is shown in fig 3.(a), we have observed that the codec G.711 and G.726ar24 has less end-to-end delay up to 150 nodes and at 250 nodes, it is observed that G.729 has less end-to-end delay. In mobile WiMAX, which is shown in fig 3.(b), we have observed that at 250 nodes, the codec G.726ar24 has less end-to-end delay whereas at 150 nodes, G.711 has maximum end-to-end delay because of the mobility and increase in the number of nodes in the network.

\subsection{Throughput}

The number of packets successfully delivered over a network is called as Throughput. It is measured as bits/second or bytes/second [25]. The Throughput, $\mathrm{T}$ in bits/sec is as per the following:

$$
\mathrm{T}=\frac{\text { Total bytes received } * 8}{\mathrm{t} 2-\mathrm{t} 1}
$$

Where, $t 1$ is time at which the first packet is received and $t 2$ is time at which the last packet is received.

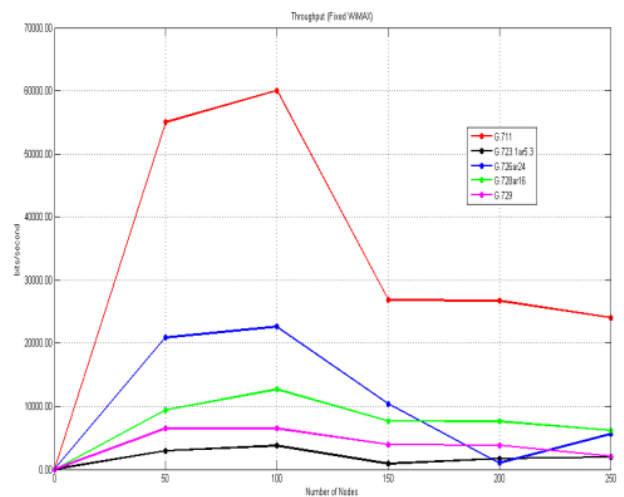

Fig.4. (a) Throughput of FixedWiMAX with respect to number of nodes

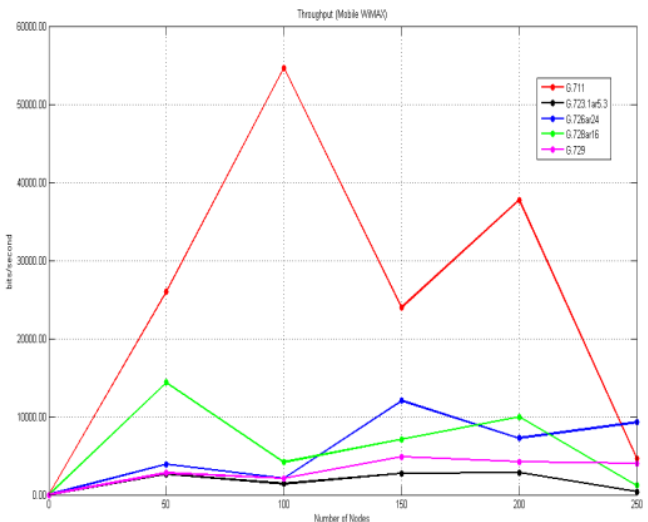

Fig.4. (b) Throughput of MobileWiMAX with respect to number of nodes 
In both fixed and mobile WiMAX (i.e. fig 4.(a) and fig 4.(b)), we have observed that the codec G.711 has maximum throughput compared to other codecs. G.711 uses logarithmic compression while transmitting the packets, and also offers bit rate of 64 Kbits for transmission, because of this feature G.711 is used in wide range of application.

\subsection{Average Jitter}

Average jitter is the amount of variation in the arrival of packets to the destination $[26,27]$. The value of jitter is calculated from end-to-end delay. The average Jitter, $J$ in seconds is as follows:

$$
\mathrm{J}=\frac{\text { Total packet jitter for all received packets }}{\text { number of packets received }-1}
$$

Packet Jitter $=\mathrm{t} 2-\mathrm{t} 1$

Where $t 2$ is transmission delay of current packet and $t 1$ is transmission delay of previous packet.

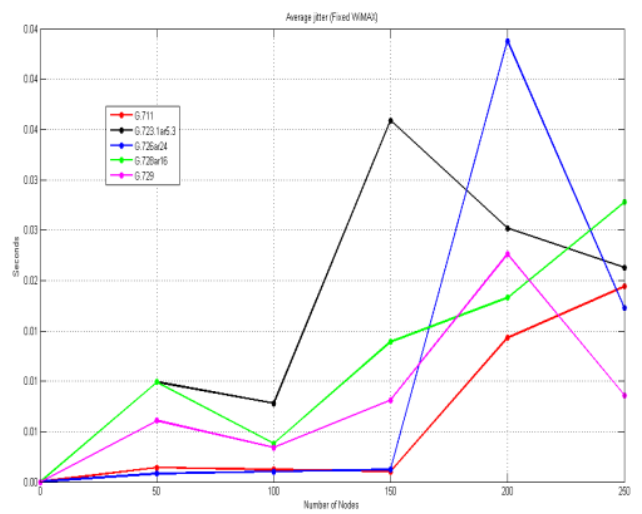

Fig.5. (a) Average jitter of FixedWiMAX with respect to number of nodes

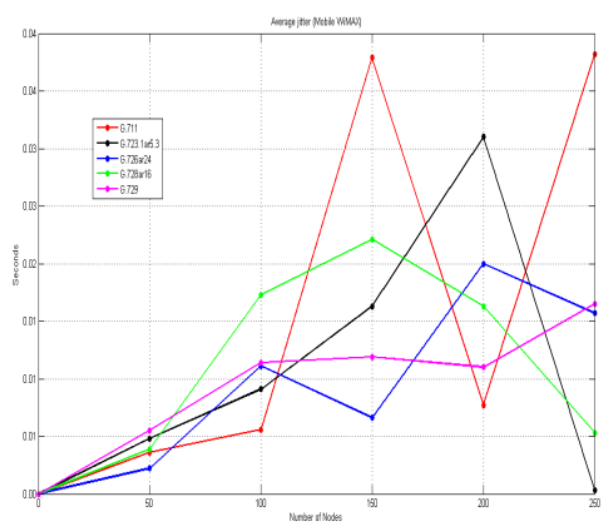

Fig.5. (b) Average jitter of MobileWiMAX with respect to number of nodes 
From fig 5.(a), we have observed that codec G.711 has less jitter up to 200 nodes and from fig 5.(b),we haveobserved that codec G.723.1ar5.3 has less jitter. The codec G.711 is used by the PSTN because it works well with fixed assets, but when applied on mobile WiMAXnetwork the results are not satisfactory.

\subsection{Average one-way delay}

One-way delay is equivalent to the difference between arrival time at the destination and departure time at the source. One-way delay is measured in terms of seconds. The one-way delay, D` in seconds is as follows:

$$
D(a, b)=t 1(a)-t 2(b)
$$

Where $\mathrm{a} \& \mathrm{~b}$ are destination and source node respectively, $\mathrm{t} 1$ is the arrival time at node $\mathrm{a}$ and $\mathrm{t} 2$ is the departure time at node $b$.

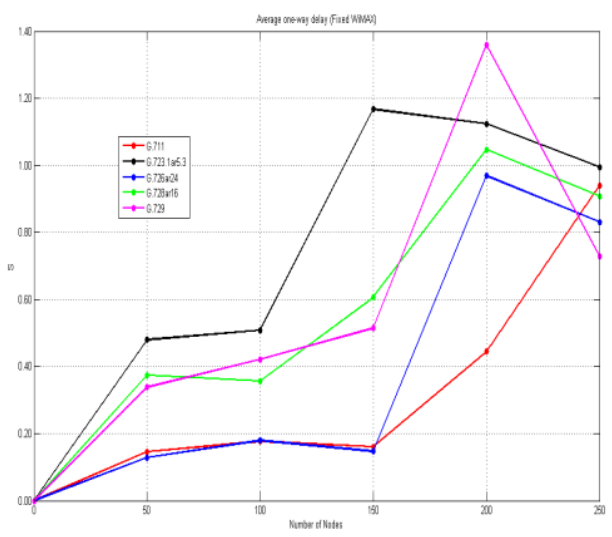

Fig.6. (a) Average one-way delay of Fixed WiMAX with respect to number of nodes

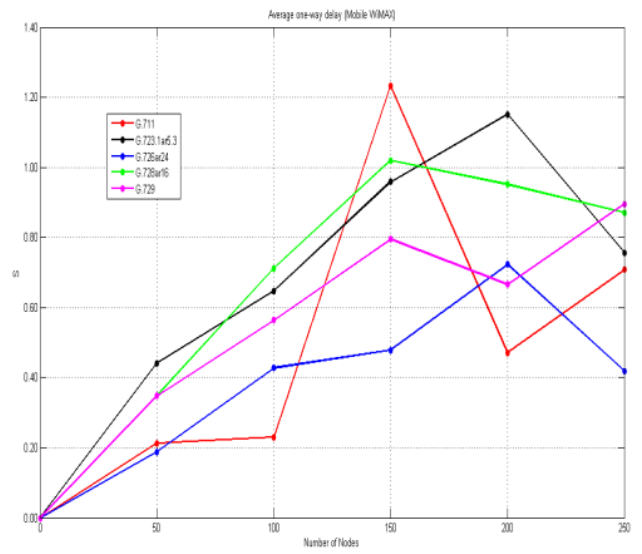

Fig.6. (b) Average one-way delay of MobileWiMAX with respect to number of nodes 
From Fig 6.(a) and fig 6.(b) we observed that the codec G.711 provides less one-way delay as compared to other codecs because G.711 works well with the fixed network environment. Whereas in case of Mobile WiMAX, at 250 nodes, the G.726ar24 provides less one-way delay compared to other codecs because it uses $24 \mathrm{Kbps}$ bit rate for transmission and is majorly used in cordless telephony wireless phones.

\subsection{Average MOS (Mean Opinion Score)}

Mean Opinion Score gives VoIP testing a number value as an indication of perceived quality of received voice after being transmitted and compressed using codecs. Different scores are shown in Table 4 [6].

Table 4. Mean Opinion Scores

\begin{tabular}{ll}
\hline Quality Scale & Score \\
\hline Excellent & 5 \\
Good & 4 \\
Average & 3 \\
Poor & 2 \\
Bad & 1 \\
\hline
\end{tabular}

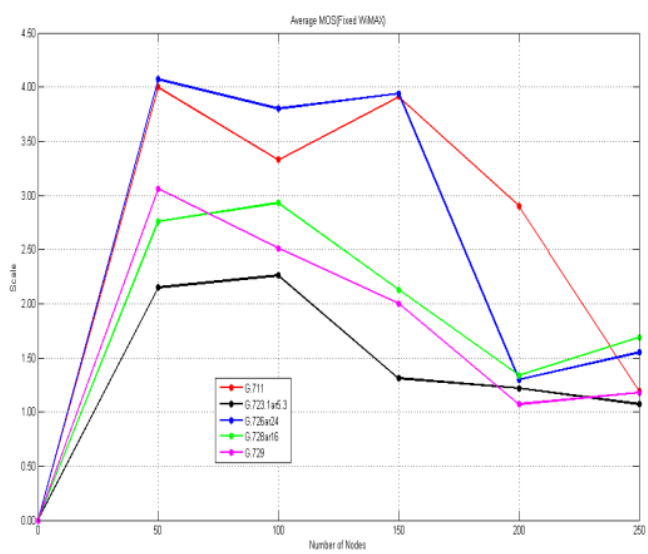

Fig.7. (a) Average MOS of FixedWiMAX with respect to number of nodes

From fig 7.(a), we observed that the codec G.726ar24 provides high MOS compared to other codecs, whereas in case of mobile WiMAX(i.e. fig 7.(b)) both G.711 and G.726ar24 provide high MOS.This is because codec G.711 has voice sampling rate of $8 \mathrm{kHz}$ and each sample is encoded with 8 bits resulting in a constant 64kbps bit rate and offers a very good voice quality. 


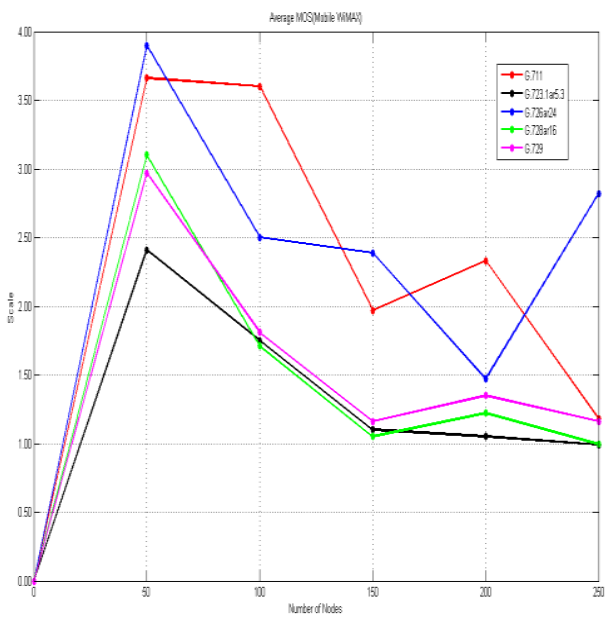

Fig.7. (b) Average MOS of MobileWiMAX with respect to number of nodes

\section{Conclusions}

The two WiMAX network (i.e. fixed and mobile WiMAX) are compared with the various VoIP codecs, such asG.711, G.723.1ar5.3, G.726ar24, G.728ar16 and G.729 by increasing the number of nodes up to 250 in each network. From the obtained result, we conclude that in fixed WiMAX the codec G.711 provides high throughput, less end-to-end delay, less jitter, less one-way delay and codec G.726ar24 provides high MOS. In mobile WiMAX, codec G.726ar24 provides less end-to-end delay, less one-way delay and high MOS, codec G.723.1ar5.3 provides less jitter and G.711 provides high throughput compared to all codecs. Overall G.711 provides high throughput in both fixed and mobile WiMAX networks.

\section{References}

[1] Daan Pareit, Bart Lannoo, Ingrid Moerman, and Piet Demeester, "The History of WiMAX: A Complete Survey of the Evolution in Certification and Standardization for IEEE 802.16 and WiMAX”, IEEE, 2011.

[2] Bo Li, Yang Quin, et.al, “A Survey on Mobile WiMAX”, IEEE Communication Magazine, 2007.

[3] Ioannis Papapanagiotou, Dimitris Toumpakaris, Jungwon Lee, Michael Devetsikiotis, "A Survey on Next Generation MobileWiMAX Networks: Objectives, Features and Technical Challenges”, IEEE, 2009.

[4] Sayan Kumar Ray, Krzysztof Pawlikowski, Harsha Sirisena, "Handover in Mobile WiMAX Networks: The State of Art and Research Issues", IEEE, 2010

[5] Joshi, Pranita D., and Smita Jangale. "Analysis of VoIP traffic in WiMAX using NS2 simulator." International Journal of Advanced Research in Computer Science and Electronics Engineering (IJARCSEE) 1.2 (2012): pp-109.

[6] Othman, Hazri Raziff, et al. "Performance analysis of VoIP over mobile WiMAX (IEEE 802.16 e) besteffort class." Control and System Graduate Research Colloquium (ICSGRC), 2014 IEEE 5th. IEEE, 2014.

[7] Jeffrey G. Andrews, Arunabha Ghosh, Rias Muhamed, "Fundamentals of WiMAX , Understanding Broadband Wireless Networking”, 2007. 
[8] Ali Nawaz Naqvi, Ash Mohammad Abbas, Tofik Ali Chouhan, "Performance Evaluation of Fixed and Mobile WiMAX Networks for UDP Traffic", International Journal of Advanced Research in Computer and Communication Engineering, Vol. 1, Issue.8, 2012.

[9] Mai Tran, George Zaggoulos, Andrew Nix and Angela Doufexi, "Mobile WiMAX: Performance Analysis and Comparison with Experimental Results", IEEE, 2008.

[10] Vladimir Bykovnikov, "The Advantages of SOFDMA for WiMAX”, Intel corporation, 2005

[11] M. Atif Qureshi, ArjumandYounus Muhammad Saeed, Farhan Ahmed Sidiqui , Nasir Touheed , and M. Shahid Qureshi , "Comparative Study of VoIP over WiMax and WiFi", IJCSI International Journal of Computer Science Issues, Vol. 8, Issue. 3, No. 1, 2011.

[12] Raghavendra ,Ganiga Balachandra ,Muniyal Pradeep," Characteristic Analysis of VoIP Traffic for Wireless Networks In Comparison with CBR using QualNet Network Simulator", International Journal of Computer Applications ,Volume 50, No.11, 2012.

[13] Qualnet 7.1 Multimedia and Enterprise Model Library, Scalable Network Technologies, Culver city, CA, 2013.

[14] Schulzrinne, Henning, and Jonathan Rosenberg. "A Comparison of SIP and H. 323 for Internet Telephony." Proc. International Workshop on Network and Operating System Support for Digital Audio and Video (NOSSDAV). sn, 1998.

[15] Glasmann, Josef, Wolfgang Kellerer, and Harald Müller. "Service architectures in H. 323 and SIP: A comparison." Communications Surveys \& Tutorials, IEEE 5.2 (2003): 32-47.

[16] Ali M. Alsahlany, "Performance Analysis of VoIP traffic over Integrating Wireless LAN and WAN using different Codecs.", International Journal of Wireless \& Mobile Networks (IJWMN) ,Vol. 6, No. 3, 2014.

[17] Preetinder Singh, Ravneet Kaur, "VOIP Over Wimax: A Comprehensive Review", (IJCSIT) International Journal of Computer Science and Information Technologies, Vol. 5, 2014.

[18] M.A. Mohamed, F.W. Zaki and A.M. Elfeki,"Performance Analysis of VoIP Codecs over WiMAX Networks", IJCSI International Journal of Computer Science Issues, Vol. 9, Issue.6, No.3, 2012.

[19] Tarik ANOUARI, Abdelkrim HAQIQ, "Performance Analysis of VoIP Traffic in WiMAX using various Service Classes", International Journal of Computer Applications, Volume.52, No.20, 2012.

[20] Senthilkumar L Krishna Chennakesava Rao.M, "Performance Analysis of VoIP Codecs for H323", IJCA, 2012.

[21] HiraSathu , Mohib A. Shah," Performance Comparison of VoIP Codecs on Multiple Operating Systems using IPv4 and IPv6 “, International Journal , Vol. 2, No. 2, 2012.

[22] Priyanka, Jyoteesh Malhotra, Kuldeep Sharma, "Simulative Investigation of QoS parameters for VoIP over WiMAX networks", IJCSI International Journal of Computer Science Issues, Vol. 10, Issue .2, No.3, 2013.

[23] Vikram Mehta, Neena Gupta, "Performance Analysis of QoS Parameters for Wimax Networks", International Journal of Engineering and Innovative Technology (IJEIT), Volume.1, Issue.5, 2012.

[24] Qualnet 7.1 user's guide, Scalable Network Technologies, Culver city, CA, 2013.

[25] Vimmi Malhotra, Lalit Kumar, "QOS Based Analysis in IEEE 802.11 and IEEE 802.16 Integrated Networks", IJARCSSE, Vol.3, Issue.7, 2013.

[26] D. M. Ali, K. Dimyati, "Performance Analysis of Delay Jitter in Mobile WiMAX Systems ", International Conference on Information and Electronics Engineering IPCSIT, Vol.6, 2011.

[27] J Sangeetha, Pranav B, Khan Heena, K N B Murthy, Ram P Rustagi, “An Analysis and Comparison of Different Routing Algorithms in WiMAXNeworks", IEEE IACC, 2015.

[28] J Sangeetha, Suthikshn Kumar, "A comparative study on WiFi and WiMAX networks", IEEE International Conference on Computational Intelligence and Computing Research (ICCIC), pp. 1-5, 2010 


\section{Authors' Profiles}

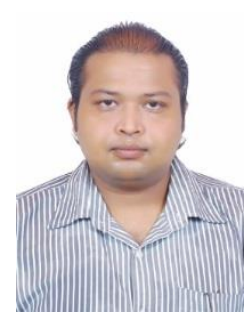

Pranav Gangadhar Balipadi presently pursuing his Bachelor of Engineering in Information Science \& Engineering, from PES Institute of Technology, Bangalore, India. His research area of interest includes Wireless Networks, Network Security and Cyber Forensics

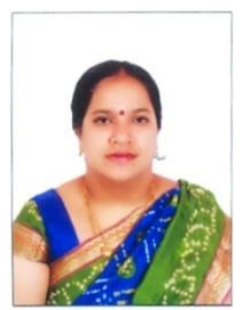

J Sangeetha received her Bachelor's degree from the Dept of Computer Science \& Engineering, from Bangalore University, India, and Master's in Information Technology from Bangalore University, India. She is pursuing her Ph.D from Visvesvaraya Technological University, Belgaum, India. She is currently working as Associate Professor, from the Department of Information Science \&Engineering at PES Institute of Technology, Bangalore, India. Her research interest includes Mobile Computing, Wireless Networks, Networks and Security and Nature Inspired Algorithms.

How to cite this paper: Pranav Gangadhar Balipadi, J Sangeetha,"An Analysis of VoIP Application in Fixed and Mobile WiMAX Networks", International Journal of Wireless and Microwave Technologies(IJWMT), Vol.6, No.5, pp.22-34, 2016.DOI: 10.5815/ijwmt.2016.05.03 